Revue d'histoire de l'Amérique française

REVUE D.HISTOIRE DE L'AMÉRIQUE FRANÇAISE

\title{
Urbanisation et population : le cas de Montréal en 1861
}

\section{Jean-Claude Robert}

Volume 35, numéro 4, mars 1982

URI : https://id.erudit.org/iderudit/304010ar

DOI : https://doi.org/10.7202/304010ar

Aller au sommaire du numéro

Éditeur(s)

Institut d'histoire de l'Amérique française

ISSN

0035-2357 (imprimé)

1492-1383 (numérique)

Découvrir la revue

Citer cet article

Robert, J.-C. (1982). Urbanisation et population : le cas de Montréal en 1861.

Revue d'histoire de l'Amérique française, 35(4), 523-535.

https://doi.org/10.7202/304010ar d'utilisation que vous pouvez consulter en ligne.

https://apropos.erudit.org/fr/usagers/politique-dutilisation/ 


\title{
URBANISATION ET POPULATION: LE CAS DE MONTREAL EN 1861
}

\author{
JEAN-CLAUDE ROBERT \\ Département d'histoire \\ Université du Québec à Montréal
}

La formation de la population est une dimension fondamentale de la croissance urbaine. Toutefois, il est habituellement très difficile d'arriver à saisir toutes les composantes de cette croissance. Les recensements du Bas-Canada nous renseignent sur les lieux de naissance des migrants internationaux, mais jamais sur la provenance des natifs du Québec. Pourtant, les déplacements internes de la population sont fort importants au XIXe siècle pour l'ensemble du territoire et non seulement pour les villes. D'une manière générale, cette mobilité géographique de la population avait été sous-estimée, mais des recherches récentes montrent la grande fluidité des mouvements internes de population ${ }^{1}$ et posent la question de leur interprétation.

À partir des listes nominatives du recensement de 1861 , ce texte propose de jeter un premier coup d'oeil sur un échantillon de migrants nés à l'intérieur du Bas-Canada, mais que l'on retrouve à Montréal à cette date ${ }^{2}$. C'est en quelque sorte un instantané: n'ayant procédé ni à des reconstitutions de famille ni à des jumelages de données, nous ignorons depuis quand ces gens sont en ville et nous ne savons pas combien de temps ils y demeureront. Notre objectif demeure modeste et nous y sommes contraint par le caractère même de nos sources; toutefois nous croyons que la rareté des données sur les migrations vers la ville au XIXe siècle justifie la démarche. Nous proposons donc une étude rapide du groupe des migrants, précédée d'une mise au point sur la croissance économique et démographique de Montréal.

\section{Montréal en 1861}

La population de la ville atteint 90323 habitants en 1861 . C'est, à l'époque, la ville la plus populeuse des colonies britanni-

1 Citons les études de Gagan sur le comté de Peel en Ontario et celles du groupe de Gérard Bouchard sur le Saguenay. En particulier voir, C. Pouyez, Raymond Roy et Gérard Bouchard, «La mobilité géographique en milieu rural: le Saguenay, 1852-1861», Histoire sociale/Social History, XIV, 27 (mai 1981): 123-155.

2 Le travail de dépouillement a été rendu possible grâce au Fonds institutionnel de recherches (UQAM) et au Projet d'Atlas historique du Canada. Nous tenons à remercier Allan Conter et Robert Nahuet pour le codage des ménages. 
ques de l'Amérique du nord. La ville de Québec ne compte que 51109 personnes et la population de Toronto, qui vient au troisième rang, atteint 44821 personnes.

Depuis 1821, la population de Montréal a presque quintuplé; cependant c'est durant la décennie qui précède le recensement de 1861 qu'elle connaît sa croissance la plus rapide. En fait, ces années marquent un seuil dans l'évolution générale de la ville; elles correspondent, en gros, à sa première industrialisation et aussi, au début de l'urbanisation des Canadiens français.

C'est que le cléveloppement rapide du Haut-Canada après 1815 donne à Montréal un hinterland important et en constante expansion. Ses fonctions de centre de commerce et de noeud de communications se développent. La croissance de ces deux fonctions donne naissance à un important secteur de fabrication: la ville tend à remplacer les importations par des productions locales lorsque cela est possible. C'est en partie ce qui explique l'essor de l'industrie de la chaussure à Montréal. Le même phénomène se retrouve dans le secteur du matériel de transport: les forges et les fonderies de la ville ont travaillé, dès les années 1820 , pour fabriquer des chaudières à vapeur et pour équiper les navires du SaintLaurent ${ }^{3}$. Plus tard, dans les années cinquante, le Grand Tronc établit ses ateliers d'entretien et de construction du matériel roulant à la Pointe Saint-Charles ${ }^{4}$.

Vers le milieu du siècle, quatre secteurs de l'économie montréalaise sont profondément touchés par une première industrialisation: la chaussure, la minoterie, le travail du fer et celui du bois. À ce moment, la ville joue le rôle de métropole économique de l'Amérique du nord britannique. Sa prééminence s'étend aussi au domaine de la finance et, même si les émeutes de 1849 lui ont fait perdre la fonction de capitale politique, ses fonctions administratives demeurent très importantes.

Ces transformations ne vont pas sans influer sur la demande de main-d'oeuvre, qu'il s'agisse d'ouvriers qualifiés ou non; la ville offre un éventail assez large de possibilités d'emploi. Par exemple, les transformations subies par le secteur de la chaussure permettent de remplacer le cordonnier qualifié par un ouvrier à qui il suffit de montrer le fonctionnement d'une machines. Soulignons aussi l'im-

3 Voir G.J.J. Tulchinsky, The River Barons (Toronto, University of Toronto Press, 1977) et W. Kilbourn, The elements combined (Toronto, Clarke, Irwin, 1960).

4 Pour une description détaillée des ateliers et des travaux, voir Montreal Business Sketches with a Description of the City of Montreal (Montreal, M. Longmore, 1865). 5 Pour l'analyse de la mécanisation de la cordonnerie à Montréal, voir Joanne Burgess, «L'industrie de la chaussure à Montréal: 1840-1870 - Le passage de l'artisanat à la fabrique", Revue d'histoire de l'Amérique française, 31,2 (septembre 1977): 187-210. 
portance des améliorations dans les transports: la morte saison diminue d'autant et cela signifie sinon la suppression du chômage saisonnier du moins sa réduction. Tous ces facteurs contribuent à rendre la demande de main-d'oeuvre plus grande et surtout plus stable dans son ensemble.

La composition de la population évolue aussi. À partir de 1831, la ville avait vu se consolider une majorité de citoyens d'origine britannique. Cette situation, qui dure jusque vers 1865 , est causée en bonne partie par une immigration en provenance de Grande-Bretagne. Toutefois, en examinant d'un peu plus près le tableau 1, qui représente les origines ethniques et le lieu de naissance de la population montréalaise, nous pouvons faire un certain nombre d'observations. La première fait voir le déclin relatif des natifs de Grande-Bretagne: formant $35 \%$ de la population en 1844, ils ne représentent plus que le quart de la population en 1861 . La seconde montre l'augmentation constante de la population née au Bas-Canada et d'origine britannique, qui passe de $13 \%$ en 1825 à $25 \%$ en 1861. Enfin, remarquons la progression constante des BasCanadiens d'origine française depuis 1844.

Poussant plus loin l'analyse de la croissance, comparons maintenant les taux annuels moyens de croissance pour les divers groupes. 


\section{TABLEAU I}

Montréal: origines ethniques et lieux de naissance

\begin{tabular}{|c|c|c|c|c|c|c|c|c|}
\hline Total & & $\begin{array}{l}\text { Bas-Canada } \\
\text { origine } \\
\text { française }\end{array}$ & $\begin{array}{l}\text { Bas-Canada } \\
\text { origine } \\
\text { britannique }\end{array}$ & $\begin{array}{l}\text { Grande- } \\
\text { Bretagne }\end{array}$ & Angleterre & Écosse & Irlande & Autres \\
\hline 1825 & 22540 & $12273-54 \%$ & $2847-13 \%$ & $6270-28 \%$ & $1249-6 \%$ & $1380-6 \%$ & $3641-16 \%$ & $1150-5 \%$ \\
\hline 1844 & 44591 & $19041-43 \%$ & $8863-20 \%$ & $15468-35 \%$ & $3161-70 \%$ & $2712-6 \%$ & $9595-22 \%$ & $1219-3 \%$ \\
\hline 1852 & 57715 & $26020-45 \%$ & $12494-22 \%$ & $17744-31 \%$ & $2858-5 \%$ & $3150-5 \%$ & $11736-20 \%$ & $1457-3 \%$ \\
\hline 1861 & 90323 & $43509-48 \%$ & $22226-25 \%$ & $21668-24 \%$ & $4293-5 \%$ & $3196-4 \%$ & $14179-16 \%$ & $2920-3 \%$ \\
\hline
\end{tabular}

Source: J.-C. Robert, Montréal 1821-1871: aspects de l'urbanisation (thèse de 3e cycle, Paris, École des hautes études en sciences sociales/Paris I, 1977), 106. 


\section{TABLEAU II}

\section{Taux annuel moyen d'accroissement des éléments de la population montréalaise}

\begin{tabular}{llcll}
\hline & $\begin{array}{l}\text { Population } \\
\text { totale }\end{array}$ & $\begin{array}{l}\text { Bas-Canada } \\
\text { origine } \\
\text { française }\end{array}$ & $\begin{array}{l}\text { Bas-Canada } \\
\text { origine } \\
\text { britannique }\end{array}$ & $\begin{array}{l}\text { Née en } \\
\text { Grande- } \\
\text { Bretagne }\end{array}$ \\
\hline $1825-1842$ & 3,48 & 1,96 & 5,97 & 5,04 \\
$1842-1844$ & 4,88 & 5,36 & 7,70 & 2,96 \\
$1844-1850$ & 1,45 & 1,75 & 2,80 & 0,33 \\
$1844-1852$ & 3,23 & 3,92 & 4,30 & 1,65 \\
$1850-1852$ & 8,63 & 10,45 & 9,09 & 8,17 \\
$1852-1861$ & 5,07 & 5,86 & 6,55 & 2,23
\end{tabular}

Source: J.-C. Robert, Montréal 1821-1871: aspects de l'urbanisation, 189.

Le taux de croissance du groupe canadien-français est supérieur à celui de la ville à partir de 1842 , ce qui confirme l'observation précédente et donne à penser que les membres de ce groupe sont attirés en plus grand nombre par la ville. Cela est sans doute plus vrai pour le groupe des Canadiens anglais, encore que dans ce cas il faille tenir compte des naissances canadiennes de couples d'immigrants. Enfin, il semble bien que la ville n'arrive pas à conserver après 1842, sa population née en Grande-Bretagne.

L'examen de ces deux tableaux nous amène à conclure à l'existence de deux mouvements migratoires qui rendent compte de l'augmentation de la population montréalaise. Le premier est bien connu, c'est le mouvement de migration en provenance des Iles britanniques. À Montréal il atteint son point culminant vers 1842 . Le second est un mouvement migratoire interne qui amène à Montréal une population née au Bas-Canada d'origine française et aussi anglaise. Entre 1852 et 1861, l'augmentation de la population née au Bas-Canada correspond à $83 \%$ de la croissance totale, ce qui indique l'importance des migrations internes pour la période postérieure à 1842 .

\section{La migration interne en 1861 et les caractères des migrants}

Le recensement de 1861 comportait une question relative au lieu de naissance de chacun des membres du ménage. Si l'on en juge par les données publiées, la réponse attendue était soit le nom de pays pour les immigrants, soit 'Bas-Canada' pour les autres. Un 
examen des listes nominatives nous a fait découvrir qu'une bonne proportion de Bas-Canadiens de naissance avait compris qu'il fallait donner un lieu précis, c'est-à-dire leur paroisse natale. Un examen rapide des différents quartiers de la ville nous a montré qu'entre $16 \%$ et $61 \%$ des natifs du Bas-Canada ont ainsi précisé leur lieu de naissance.

Pour l'ensemble de la ville, nous avons dénombré 8764 cas de migration interne, soit $9,7 \%$ de la population. Ce chiffre représente un minimum parce qu'il doit y avoir des migrants parmi ceux qui, à la question, ont répondu 'Bas-Canada'. Nous avons retenu deux quartiers, Saint-Laurent et Saint-Louis, dont nous avons étudié les migrants. Le premier renferme une faible proportion de migrants internes $(7,5 \%)$ tandis que pour le second cette proportion atteint $18,2 \%$. Soulignons de plus que la composition ethnique des deux quartiers est fort différente: Saint-Laurent est un quartier à majorité anglophone (72\%) et Saint-Louis un quartier à majorité francophone $(70 \%)$.

Le groupe étudié de migrants internes se comprose de 3281 individus et représente $13 \%$ de la population de deux quartiers. Ces migrants vivent dans 1105 ménages qui à leur tour renferment un total de 7443 personnes. L'analyse de cette population de migrants s'articule autour de quatre points: les caractères démographiques, ethniques, sociaux, ainsi que la provenance géographique. En terminant nous donnerons quelques exemples de circuits migratoires.

La première observation montre que les femmes dominent le groupe, car elles forment $54 \%$ des migrants. Ensuite nous notons la jeunesse relative de la population: l'âge moyen se situe à 30,3 ans. Précisons toutefois qu'il n'y a pas de concentration remarquable de jeunes enfants: seulement $15,6 \%$ de nos migrants appartiennent au groupe d'âge de 15 ans et moins; la proportion de cette classe d'âge dans la population urbaine est de 37,4\%. En fait, c'est un groupe où les jeunes adultes dominent: le groupe des 21-30 ans correspond au tiers de nos migrants. Enfin, une dernière observation, le statut marital: les individus sont mariés dans une proportion de près de $60 \%$.

Au plan ethnique, les migrants sont à $85 \%$ canadiens-français, mais cette proportion n'est pas nécessairement représentative de toute la migration interne à Montréal. Les migrants d'origine britannique ont bien pu s'établir en nombre relativement faible dans ces deux quartiers et préférer d'autres parties du territoire urbain.

Au plan social, nous examinerons la structure professionnelle 
du groupe des migrants. En premier lieu, notons l'importance des migrants ne déclarant aucune profession: il s'agit de près de la moitié. Même en tenant compte des enfants et des vieillards, cette proportion nous semble élevée, compte tenu de l'âge moyen des migrants. En fait, les enfants (moins de seize ans) y forment $28,6 \%$ du groupe tandis que les vieillards (plus de 65 ans) comptent pour $4,6 \%$. Ce sont les femmes qui composent la majorité $(80 \%)$ des sans-profession. Sans pouvoir aller beaucoup plus loin dans cette analyse, signalons néanmoins la possibilité d'un sous-emploi des femmes ainsi que d'un sous-enregistrement de certaines professions par le recensement. En particulier certains petits métiers ne sont presque jamais recensés: nous pensons ici, par exemple, aux porteurs des marchés et aux nombreux revendeurs et revendeuses.

L'autre moitié du groupe des migrants déclare 177 professions distinctes. Toutefois la variété n'est qu'apparente: $70 \%$ des effectifs se concentrent dans 20 professions et les six plus nombreuses regroupent tout près de la moitié des migrants déclarant une profession. Ces six sont, dans l'ordre, les domestiques $(12 \%)$, les menuisiers $(8 \%)$, les cordonniers $(8 \%)$, les couturières $(7,5 \%)$, les commis $(7 \%)$ et les journaliers $(5,6 \%)$.

Constatons l'importance des professions à faible qualification: domestiques, journaliers, cordonniers et couturières. La cordonnerie, rappelons-le, a subi une transformation profonde et, en 1861, les effectifs recensés comme 'cordonniers' ne sont plus exclusivement des coordonniers de métier. Dans une bonne mesure, nous retrouvons dans cette catégorie beaucoup d'ouvriers en cordonnerie qui, soit travaillent à la pièce en exécutant une seule opération, soit conduisent des machines; dans ces cas il s'agit de 'cordonniers' qui seraient incapables de fabriquer entièrement une chaussure. Quant à l'importance du secteur pour Montréal, précisons qu'à la fin de la décennie, on estime que $20 \%$ de la population dépend de la cordonnerie ${ }^{6}$. Pour les couturières, il s'agit d'une occupation traditionnelle apprise tôt dans la famille. C'est aussi une profession qui connaît une première mécanisation avec

6 W. J. Patterson, Statements Relating to the Trade, Navigation, Finances, etc, etc, of the Dominion of Canada and Annual Report on the Commerce of Montreal for 1868 (Montreal, Starke, 1869): «The extent of this branch of manufacture will be appreciated, when it is stated that in Montreal there are 20 factories, (5 of them small establishments) employing about 5,000 persons in the various departments, - and it is estimated that the proportion of the population dependent upon this branch of enterprise amounts to 20,000 . The improvements in machinery, introduced into the principal factories, now enable the larger firms to produce nearly 200 different kinds of Boots and Shoes. The machinery in use includes - 250 sewing machines, 50 pegging machines, 30 closing machines, 15 soles-sewing machines, 20 sole cutters, - beside machinery for eyeletting, punching, skining, rolling, etc.», 15 . 
l'introduction de la machine à coudre bon marché ${ }^{7}$. La profession de menuisier est également une profession plus traditionnelle, dont les rudiments sont souvent appris à la campagne. Cependant, notons qu'à côté de ces professions, on en retrouve d'autres exigeant une plus grande qualification, comme celles de commis, marchand et avocat.

Nous ne voyons pas se dessiner de différences marquées au plan de l'ethnicité sauf en ce qui concerne la profession de commis. Près de $20 \%$ des anglophones déclarant une profession sont commis. Toutefois, même en défalquant du nombre les anglophones, le groupe des commis reste l'un des six premiers pour les Canadiens français.

Examinons maintenant les professions des chefs de ménage qui sont eux-mêmes migrants. L'analyse des 685 cas fait apparaître également une concentration marquée, alors que $68 \%$ des effectifs des chefs de ménage se répartissent dans 18 professions. Seulement 14 de celles-ci sont au nombre des 20 professions les plus fréquentes pour l'ensemble des migrants. Les professions importantes qui disparaissent dans le groupe des chefs de famille sont celles qui correspondent à des professions de jeunes: domestique, étudiant, apprenti, etc. Un seul chef de ménage est domestique. Dans d'autres cas, comme celui des couturières ${ }^{8}$, il s'agit de professions exercées en bonne partie par des célibataires ou des personnes seules.

La relation entre l'âge et la structure professionnelle de l'ensemble des migrants fait apparaître d'autres caractères. D'abord la jeunesse du groupe est soulignée par le fait que plus de la moitié des migrants déclarant une profession sont dans la classe d'âge des 16-30 ans. Mais si nous étudions d'un peu plus près les 10 professions les plus nombreuses, nous voyons apparaître trois catégories de professions: celles où les jeunes dominent, celles où les travailleurs plus mûrs forment la majorité et celles où l'âge semble indifférent. Les domestiques appartiennent à la première catégorie avec $35 \%$ de leurs effectifs dans la classe d'âge des moins de 21 ans. C'est décidément une profession de jeunes, puisque les trois quarts des domestiques n'ont pas 30 ans. Les charretiers appartiennent à la seconde catégorie: $80 \%$ de leurs effectifs ont plus de 30 ans. Contrairement à la profession de domestique, occupation de

\footnotetext{
Martha E. Brent, «A stich in Time: The Sewing Machine Industry of Ontario, 1860-1897», Material History Bulletin/Bulletin d'histoire de la culture matérielle, 10 (Spring 1980): 1-31.

${ }_{8}$ C'est le cas en particulier aux États-Unis: «The markets of seamstresses were especially crowded with unmarried and widowed women, not to speak of those whose husbands were "travelling» in the informal divorce procedure of the day». David Montgomery, "The working classes of the pre-industrial city», R. A. Mohl and N. Betten, ed., Urban America in Historical Perspective (New York, Weybright and Telley, 1970), 109.
} 
nature souvent transitoire et urbanisante, celle de charretier pourrait représenter une profession de 'fin de carrière', à laquelle on accède après avoir accumulé un minimum de capital pour s'acheter voiture et cheval $^{9}$. Enfin, dans la troisième catégorie nous retrouvons les couturières, les cordonniers et les commis. La répartition des âges y est plus équilibrée; le groupe des moins de 21 ans y est important, sans être dominant.

L'incidence de la distribution des professions selon le sexe montre, comme on pourrait s'y attendre pour l'époque, un déséquilibre prononcé. D'abord au plan des effectifs, les migrants déclarant une profession sont des hommes dans une proportion de $73 \%$. Ensuite, il y a le très fort degré de concentration des femmes à l'intérieur de quelques professions, puisque $75 \%$ des femmes travaillent dans trois domaines: $38 \%$ sont domestiques, $28 \%$ couturières et 5,6\% modistes. Les autres professions sont dans les secteurs traditionnellement occupés par les femmes: travail de maison, services de santé, enseignement. L'importance de la profession de domestique est à souligner car les femmes constituent $90 \%$ des effectifs de cette profession chez nos migrants (hommes et femmes).

Avant de passer aux origines géographiques, reprenons les grandes conclusions qui ressortent de l'analyse du groupe des migrants. Il s'agit d'abord d'un groupe où les femmes sont majoritaires; c'est aussi un groupe caractérisé par sa jeunesse et son appartenance ethnique canadienne-française. Sa structure professionnelle est très concentrée et correspond en gros aux caractères d'une population récemment urbanisée, c'est-à-dire des professions de nature transitoire et des professions à faible niveau de qualification. Enfin les femmes sont moins présentes dans cette structure professionnelle et leur choix, davantage étroit.

L'origine géographique de nos migrants se répartit dans 266 endroits différents, correspondant, pour la plupart, à des noms de paroisse. Nous avons réussi à classer la presque totalité de ces lieux et les avons regroupés en sept zones: les villes (essentiellement Québec, Trois-Rivières, et Sherbrooke), l'île de Montréal, les comtés entourant l'île, ceux de la vallée du Richelieu, les comtés riverains du Saint-Laurent entre Berthier et Québec, les Cantons de l'est et enfin tous les comtés situés à l'est de Québec.

L'importance de la région montréalaise ressort car près de $70 \%$ des migrants sont nés dans les paroisses rurales de l'île de

Ce qui sera moins le cas après l'introduction des grosses entreprises de transport possédant parc de voitures et écuries. Voir Margaret Heap, «La grève des charretiers à Montréal, 1864», RHAF, 31,3 (décembre 1977): 371-395. 
Montréal (9\%) et dans les comtés limitrophes (60\%). Les villes viennent ensuite, particulièrement Québec qui, à elle seule, fournit $13 \%$ des effectifs. Ensuite nous retrouvons la vallée du Richelieu avec près de $10 \%$ de migrants et la région entre Berthier et Québec, $5,2 \%$. Les Cantons de l'est ne comptent que pour 2,7\% et les comtés au-delà de Québec à peine $1 \%$.

Voilà donc les contours du bassin démographique de Montréal si nous excluons les migrations transatlantiques et celles, peu nombreuses, en provenance des autres colonies britanniques de l'Amérique. C'est le cas classique d'une ville qui recrute sa population dans son voisinage immédiat, surtout à une époque où le réseau ferroviaire en est à ses débuts. Notons cependant quelques traits particuliers. D'abord la prédominance des paroisses de la rive nord du Saint-Laurent: presque toutes les paroisses entre l'Outaouais et Berthier sont représentées y compris des paroisses de colonisation récente. Ensuite, soulignons le cas de Québec, qui arrive bon premier comme lieu d'origine des migrants. Près de la moitié des migrants anglophones sont natifs de cette ville. Cela ne saurait trop surprendre quand on pense au rôle de port d'entrée joué par Québec pour l'immigration britannique. De plus, après 1850 , l'économie de cette ville semble moins dynamique, ce qui expliquerait le nombre de migrants tant anglophones que francophones.

Nous n'avons pas trouvé de relation significative entre les lieux de naissance et la profession. D'abord la répartition géographique des migrants déclarant une profession est comparable à celle des migrants sans profession. Ensuite, concernant des spécialités professionnelles locales, signalons que $20 \%$ des effectifs des commis sont nés à Québec et que $13 \%$ de nos cordonniers sont nés à Lavaltrie. Néanmoins, cela reflète davantage des liens entre les migrants d'un même endroit qu'une spécialisation locale.

Nous aimerions attirer l'attention sur un dernier phénomène, les circuits migratoires. En effet la migration n'est pas nécessairement un déplacement unique, de l'endroit de la naissance à Montréal par exemple. Ainsi, les migrants repérés au recensement de 1861 peuvent fort bien n'être pas venus directement à Montréal pour y rester toute leur vie. Néanmoins ces mouvements sont très difficiles à suivre, à moins que l'on ne dispose d'un journal comme dans le cas de la famille Benson étudiée par Michael Katz dans son étude sur Hamilton ${ }^{10}$. Cependant, les lieux de naissance des mem-

10 Michael Katz, The People of a Hamilton, Canada West (Cambridge, Harvard University Press, 1975). 
bres d'une même famille peuvent servir d'indicateur de ce genre de circuit. Précisons tout de suite qu'il ne s'agit que d'un indicateur grossier et qu'en aucun cas nous pouvons considérer ces circuits migratoires comme définitifs. Tous les couples n'ont pas eu un enfant à chacun des endroits où ils ont vécu et, de plus, nous ignorons tout des déplacements des célibataires. Les quelques cas que nous présentons ici n'ont pas d'autre prétention que d'indiquer la présence de cette modalité de migration.

Pour $10 \%$ de nos 1105 ménages, les lieux de naissance des membres de la famille permettent de conclure qu'ils ont connu ce genre de déplacement. Nous avons isolé quatre types généraux de circuits. Le premier est celui des immigrants britanniques qui se sont installés successivement dans le Haut ou le Bas-Canada. Le deuxième type correspond à des migrations à l'intérieur du BasCanada, le troisième type à des séjours dans le Haut-Canada et le dernier, aux États-Unis. Voici quelques cas.

J. McLoughlin, crieur public, est âgé de 45 ans. Lui et sa femme sont nés en Irlande. L'aînée, âgée de 20 ans, a vu le jour en Jamaïque; leur premier fils, 17 ans, est né à Québec et les quatre dernières filles, âgées respectivement de 12 ans, 10 ans, 9 ans et 6 ans, sont nées à Montréal.

La famille de François Demers a connu une expérience différente. Le père, menuisier âgé de 39 ans, est né à Beauport et la mère (34 ans) à Saint-Vincent de Paul. Leur première fille, 16 ans, naquit à Québec, la seconde, 12 ans, à Saint-Nicolas. Suivent deux fils, âgés de 10 et 9 ans, nés à Québec et deux autres fils, 8 et 3 ans, qui sont nés à Montréal.

Les déplacements vers le Haut-Canada impliquent des anglophones et des francophones. Prenons l'exemple de la famille Gassett. Le père 28 ans, caissier, est né à Québec et la mère à Picton dans le Haut-Canada. Leur fils de 6 ans, est né à Hamilton et leur fille de 2 ans, à Ingersoll. La famille d'Auguste Rochon a aussi vécu à Hamilton. Menuisier natif de Montréal, il est âgé de 47 ans et sa femme, elle aussi de Montréal, est âgée de 45 ans. Les deux aînés âgés de 22 et 20 ans, tous deux brossiers, sont nés à Hamilton. Leur première fille, 16 ans, née à Montréal, travaille avec ses frères. Pierre, le troisième fils, âgé de 14 ans et né à SainteMartine, partage le même métier. Les quatre derniers enfants, âgés de $11,8,5$ et 3 ans, sont tous nés à Montréal.

$\mathrm{Au}$ XIXe siècle les migrations vers les États-Unis sont fort communes. Quelquefois, les gens revenaient; c'est le cas de la famille de Joseph Lavoie. Cordonnier âgé de 35 ans, il est né à 
l'Assomption; sa femme, 31 ans, est née à Saint-Constant. Leur premier fils, 14 ans, est né à Montréal et le second, 12 ans, à Chicago. Leur première fille, 9 ans, est née à Montréal. Suivent deux filles et un garçon ( 7 ans, 5 ans et 3 ans), tous nés à Chambly. Enfin la petite dernière, Marie, 1 an, est née à Montréal.

Ces quelques exemples donnent une idée de la mobilité géographique même s'ils sous-estiment sérieusement le nombre d'endroits où un couple a pu s'établir successivement. Même si nous sommes conscient de la fragilité des sources et de leur caractère incomplet, il a paru important de souligner cette modalité particulière des migrations.

\section{Conclusion}

Grâce à l'ambiguïté d'une question du recensement de 1861, nous avons pu étudier les grands traits d'un échantillon de la population montréalaise de 1861 , représentant une partie de la migration interne au Québec. À cette étape de la recherche, nos conclusions ne peuvent qu'être provisoires, compte tenu de la source utilisée. Cependant nous croyons que ce pan mal connu de l'expérience collective des Montréalais méritait d'être ramené à la lumière du jour et porté à l'attention des chercheurs.

Les migrants identifiés en 1861 possèdent certaines caractéristiques précises. C'est d'abord un groupe jeune, formé de femmes pour un peu plus de la moitié. La structure professionnelle est marquée par une concentration relative et aussi par l'importance des professions à faible niveau de qualification. Au plan géographique, nous avons affaire à des migrations à courte distance, la région immédiate de Montréal fournissant près de $70 \%$ des migrants. Cependant les migrants en provenance de la ville de Québec $(13 \%)$ ) forment une exception à cette règle. Enfin, quelques cas de circuits migratoires nous permettent de voir que la migration interne n'est pas nécessairement un déplacement unique et qu'il existe un phénomène de migration par étapes.

L'étude des migrations s'inscrit dans un contexte plus vaste, celui de l'évolution socio-économique du Québec au XIXe siècle. Plus précisément nous abordons le thème de l'urbanisation de la population. Nous avons vu que ce mouvement intéressait les natifs $\mathrm{du}$ Bas-Canada, qu'ils soient anglophones ou francophones. Manifestement, dès le milieu du siècle la ville de Montréal attire les campagnards. Malgré cela, Montréal n'arrive pas à rivaliser avec d'autres pôles d'attraction, particulièrement avec les villes des États-Unis. Comme point de comparaison, l'émigration nette du Québec vers les États-Unis s'élève à 70000 personnes pour la seule 
décennie de $1850-1860^{11}$. Une croissance économique plus soutenue explique ce cas et aussi sans doute, des besoins différents de main-d'oeuvre. Les migrants se dirigent là où ils espèrent trouver de l'emploi qui corresponde tant à leur niveau de qualification qu'à leurs habitudes de travail. Le groupe canadien-français recherchait de l'emploi compatible avec son expérience de travail en milieu rural et aussi avec son habitude du travail familial. Ce dernier trait a été identifié dans plusieurs villes américaines, qu'il s'agisse de Cohoes, de Manchester ou de Lowell ${ }^{12}$. D'ailleurs, dès que les villes du Québec ont offert ce genre d'emploi, la réponse a été immédiate. En d'autres termes, l'urbanisation des Canadiens français serait conditionnée par la demande de main d'oeuvre et cette demande s'effectue dans un contexte géographique où se retrouvent des pôles d'attraction concurrents. Dans ce sens, on pourrait penser que cette urbanisation doit être étudiée non seulement dans l'aire du Québec, mais à l'échelle de toute la région comprise entre la vallée du Saint-Laurent et celle de l'Hudson. Le phénomène gagnerait ainsi à être analysé dans une perspective globale, de part et d'autre d'une frontière qui n'arrêterait guère les individus et les familles.

11 Yolande Lavoie, «Les mouvements migratoires des Canadiens entre leur pays et les États-Unis au XIXe et au XXe siècles: étude quantitative», H. Charbonneau, dir., $L a$ population du Québec: études rétrospectives (Montréal, Boréal Express, 1973), 78.

12 Daniel J. Walkowitz, Worker City, Company Town. Iron and Cotton-Worker Protest in Troy and Cohoes, New York 1855-84 (Urbana, University of Illinois Press, 1978); Tamara K. Hareven and R. Langenbach, Amoskeag, Life and Work in an American Factory City (New York, Pantheon Books, 1978); Frances H. Early, "The FrenchCanadian Family Economy and Standard of Living in Lowell, Massachusetts, 1870». Communication présentée au congrès de la Société historique du Canada, Montréal, juin 1980. 